\title{
Symptom report in detecting breast cancer-related lymphedema
}

This article was published in the following Dove Press journal:

Breast Cancer: Targets and Therapy

15 October 2015

Number of times this article has been viewed

Mei R Fu'

Deborah Axelrod ${ }^{2,3}$

Charles M Cleland'

Zeyuan $\mathrm{Qiu}^{4}$

Amber A Guth ${ }^{2,3}$

Robin Kleinman ${ }^{2}$

Joan Scagliola ${ }^{2}$

Judith Haber'

'College of Nursing, New York University, ${ }^{2}$ Department of Surgery, NYU School of Medicine, ${ }^{3} \mathrm{NYU}$ Clinical Cancer Center, New York, NY, ${ }^{4}$ Department of Chemistry and Environmental Science, New Jersey Institute of Technology, Newark, NJ, USA
Correspondence: Mei R Fu

College of Nursing, New York University,

433 First Avenue, Fourth Floor,

Room 424, New York, NY 10010, USA

$\mathrm{Tel}+\mathrm{I} 2129985314$

Emailmf67@nyu.edu
Abstract: Breast cancer-related lymphedema is a syndrome of abnormal swelling coupled with multiple symptoms resulting from obstruction or disruption of the lymphatic system associated with cancer treatment. Research has demonstrated that with increased number of symptoms reported, breast cancer survivors' limb volume increased. Lymphedema symptoms in the affected limb may indicate a latent stage of lymphedema in which changes cannot be detected by objective measures. The latent stage of lymphedema may exist months or years before overt swelling occurs. Symptom report may play an important role in detecting lymphedema in clinical practice. The purposes of this study were to: 1) examine the validity, sensitivity, and specificity of symptoms for detecting breast cancer-related lymphedema and 2) determine the best clinical cutoff point for the count of symptoms that maximized the sum of sensitivity and specificity. Data were collected from 250 women, including healthy female adults, breast cancer survivors with lymphedema, and those at risk for lymphedema. Lymphedema symptoms were assessed using a reliable and valid instrument. Validity, sensitivity, and specificity were evaluated using logistic regression, analysis of variance, and areas under receiver operating characteristic curves. Count of lymphedema symptoms was able to differentiate healthy adults from breast cancer survivors with lymphedema and those at risk for lymphedema. A diagnostic cutoff of three symptoms discriminated breast cancer survivors with lymphedema from healthy women with a sensitivity of $94 \%$ and a specificity of $97 \%$ (area under the curve $=0.98$ ). A diagnostic cutoff of nine symptoms discriminated at-risk survivors from survivors with lymphedema with a sensitivity of $64 \%$ and a specificity of $80 \%$ (area under the curve $=0.72$ ). In the absence of objective measurements capable of detecting latent stages of lymphedema, count of symptoms may be a cost-effective initial screening tool for detecting lymphedema.

Keywords: screening, risk, swelling, pain, heaviness, sensitivity

\section{Introduction}

Breast cancer-related lymphedema (hereafter, lymphedema) is a syndrome of abnormal swelling coupled with multiple symptoms resulting from obstruction or disruption of the lymphatic system associated with cancer treatment (eg, axillary surgery and/or radiotherapy). ${ }^{1-6}$ Breast cancer survivors with lymphedema in the ipsilateral upper extremity report experiencing multiple symptoms concurrently; these symptoms include selfreported arm swelling, heaviness, tightness, firmness, pain, numbness, stiffness, and impaired limb mobility. ${ }^{5-7}$ Research has demonstrated that with increased number of symptoms reported, breast cancer survivors' limb volume increased. ${ }^{6}$ Limb volume changes (LVC) as detected by the infrared perometer have significantly increased as breast cancer survivors' reports of arm swelling, heaviness, tenderness, firmness, 
tightness, and aching have increased. ${ }^{6}$ On an average, breast cancer survivors reported 4.2 symptoms for survivors with $<5.0 \%$ LVC, 5.5 symptoms for $5.0 \%-9.9 \% \mathrm{LVC}$, 7.0 symptoms for $10.0 \%-14.9 \% \mathrm{LVC}$, and 12.5 symptoms for $>15 \%$ LVC $(P<0.001){ }^{6}$

Objective measures of the limb size or limb volume have been considered a useful way in assessing and detecting lymphedema due to its quantifiable nature. However, lymphedema symptoms may indicate a latent stage of lymphedema in which changes cannot be detected by objective measures. ${ }^{8,9}$ The latent stage of lymphedema may exist months or years before overt swelling occurs. ${ }^{9}$ Symptom report may play an important role in detecting lymphedema in clinical practice. Researchers have taken efforts to develop valid and reliable instruments to assess lymphedema symptoms, that is, if an instrument (a scale or checklist) is able to assess symptoms that are true for lymphedema and if the instrument is reliable (consistent or stable) to assess lymphedema symptoms. ${ }^{8,10-12}$ A systematic review on lymphedema symptoms in detecting lymphedema was not possible due to the limited literature in this field. Thus, we undertook the effort to evaluate whether self-report of lymphedema symptoms is valid, that is, whether the symptom report can accurately (sensitivity and specificity) detect and differentiate lymphedema defined by $>200 \mathrm{~mL}$ limb volume in the affected limb using a sequential circumferential tape measurement.

\section{Purpose of the study}

The purposes of this study were to: 1) examine the validity, sensitivity, and specificity of symptoms for detecting breast cancer-related lymphedema defined by $>200 \mathrm{~mL}$ limb volume in the affected limb when comparing the unaffected limb using sequential circumferential arm measurements and 2) determine the best clinical cutoff point for the count of symptoms that maximized the sum of sensitivity and specificity for detecting breast cancer-related lymphedema defined by $>200 \mathrm{~mL}$ limb volume in the affected limb when comparing the unaffected limb.

\section{Methods}

\section{Ethical consideration}

This study was approved by the Institutional Review Board of a metropolitan medical center in New York City, USA.

\section{Recruitment}

Researchers were oriented and trained to the procedure for obtaining informed consent and collecting data. After the institutional review board approved the study, the study invitation was distributed to patients by the physicians, nurses, and lymphedema therapists who cared for the patients and to the healthy female adults living in the communities in New York City. If a patient or a healthy female adult was interested in the study, the potential participant would notify the researchers. The researchers then met with all patients and healthy female adults who responded to the study invitation to confirm the study criteria and provide a detailed explanation of the study. Participants were informed that their participation was voluntary, anonymous, and confidential. Participants were also assured that they had the right to withdraw from the study at any time without any penalty or effect on their current treatment. Written consent to the study was obtained from each participant.

\section{Research design and participants}

This cross-sectional study employed a contrast-group and purposive sampling method to recruit 250 adult female participants with different representations of lymphedema from a metropolitan cancer center and communities in the metropolitan area of New York City in 2010-2011. The inclusion criteria for participating in the study were: 1) 18 years of age or older and 2) able to read and write in English language. The exclusion criteria were: 1) the presence of serious mental disorder; 2) the occurrence of tumor metastasis; and 3) hereditary lymphedema.

Participants were divided into three groups: healthy female adults, breast cancer survivors with lymphedema, and those at risk for lymphedema. We selected healthy female adults without a history of breast cancer and lymphedema to serve as a healthy comparison group, since the majority of breast cancer survivors are female adults. We asked healthy adult female participants to consider their dominant arm as the affected arm, since women report using their dominant arm to lift heavy objects, do manual and laborious work, as well as play tennis or other sports.

We recruited breast cancer survivors who had no lymphedema diagnosis and were never treated for lymphedema as the at-risk group for lymphedema since women treated for lymphedema are at lifetime risk for lymphedema. ${ }^{8,10}$ Breast cancer survivors at risk for lymphedema must have completed surgical treatment within prior 5 years of the study enrollment since lymphedema often develops $1-5$ years after cancer treatment. ${ }^{10}$

We hypothesized that at-risk breast cancer survivors would have significantly fewer lymphedema symptoms or different symptom profiles in comparison with breast cancer survivors with lymphedema. 
Breast cancer survivors with lymphedema served as the known lymphedema group. Breast cancer survivors with lymphedema were excluded if they: 1) had lymphedema prior to breast cancer treatment; 2) had no medical record of lymphedema diagnosis; and 3) had $<200 \mathrm{~mL}$ limb volume difference in the affected limb comparing with the unaffected limb using sequential circumferential arm measurements. We hypothesized that survivors with lymphedema would have significantly more lymphedema symptoms or different symptom profiles than healthy women and at-risk survivors.

\section{Measures and instruments Demographic and medical information}

We used a structured interview tool to gather demographic, medical, and clinical information regarding breast cancer and lymphedema diagnosis, stage of diseases, cancer and lymphedema location, type of adjuvant therapy, and treatment complications. ${ }^{7,10}$ Medical records were reviewed to verify the information.

\section{Lymphedema status}

Lymphedema status was ensured by 1) self-report that was verified via medical record review to confirm that the participants had a medical diagnosis of lymphedema and were treated for lymphedema at least 6 months prior to the enrollment of the study and 2) confirmed by the researchers using sequential circumferential arm measurements that the participants had $>200 \mathrm{~mL}$ difference in limb volume when comparing the affected and unaffected limbs. ${ }^{8}, 16$ A sequential circumferential tape measurement protocol established by Armer et al and used previously by the authors was used. ${ }^{8} 16$ The same two researchers were trained, and they completed the sequential circumferential tape measurement using a flexible, noncompliant woven Juzo tape measure on both the ipsilateral and contralateral limbs of all the participants.

\section{Breast cancer and lymphedema symptom experience index}

A two-part, 34-item, 5-point Likert-type interview or selfreport instrument measured the presence of symptoms related to breast cancer and lymphedema and distress from the symptoms. Part I assessed 24 symptoms associated with breast cancer-related lymphedema, while Part II evaluated different dimensions of symptom distress. Part II of the instrument was not used in the study since symptom distress was not the focus of the study. The instrument demonstrated high internal consistency with a Cronbach's alpha coefficient of $0.92 .^{7,10-12}$ Convergent validity was demonstrated by significant correlations with dimensions of symptom distress $(r=0.35-0.93)$, including functional, emotional and psychological, attributive, sexual, and sleep problems. The breast cancer and lymphedema symptom experience index (BCLE-SEI) was able to distinguish breast cancer survivors with and without lymphedema in terms of symptom occurrence and distress $(P<0.05) .{ }^{11,12}$

Part I of the instrument was used in the study to assess 24 lymphedema symptoms of impaired limb mobility, arm swelling, breast swelling, chest wall swelling, heaviness, firmness, tightness, stiffness, numbness, tenderness, pain, aching, soreness, stiffness, redness, blistering, burning, stabbing, and tingling (pins and needles). A response frame of last 3 months was used for all participants to ensure the chronicity of symptom presence. Each item can be rated on a Likert-type scale from 0 (no presence of a given symptom) to 4 (greatest severity of a given symptom). Higher scores indicate the presence of more severe symptom. In this study, each symptom was treated as a categorical variable (ie, the presence and absence of a given symptom) and the overall count of symptoms as a continuous variable, with absolute 0 indicating the absence of any symptoms and 24 denoting maximal presence of 24 symptoms.

\section{Data collection}

Researchers were trained to perform the sequential circumferential arm measurements according to the protocol. ${ }^{8,16}$ Participants completed the instruments, including demographic information and the Breast Cancer and Lymphedema Symptom Experience Index. Researchers collected and verified the medical information by retrieving information from electronic medical records.

\section{Data analysis}

All statistical tests were conducted at the 0.05 alpha level, and all estimates included a 95\% confidence interval (CI). Descriptive statistics using SPSS version 20.0 (IBM Corporation, Armonk, NY, USA) were calculated to describe the characteristics of the participants as well as chi-squared tests for contingency tables and one-way analysis of variance for continuous variables were calculated to compare study groups on demographic and clinical characteristics. Bivariate odds ratios were computed to estimate the strength of association between symptoms and lymphedema. For symptom profiles, among the 24 symptoms, the symptoms of breast and chest wall swelling have been found to have no association with arm lymphedema and LVC, which is consistent with previous 
research. ${ }^{4,6,7,11,12}$ Thus, we only used 22 symptoms for data analysis in this study.

To test the validity of using symptom report for detection of lymphedema, that is, the ability of lymphedema symptoms to differentiate the study groups, we compared the three study groups on count of symptoms reported using one-way analysis of variance and Tukey multiple comparisons of means for statistical significance. In these comparisons, we did not assume equal variances across the three study groups. ${ }^{17}$ Fisher's exact test followed by Bonferroni adjustment was used for controlling familywise error rate for multiple comparisons of individual lymphedema symptoms among the three groups. The Bonferroni critical value indicates $P<0.002$ to be significant for the multiple comparison with 22 tests of each comparison of individual symptoms among the three groups.

Since there are no existing data to support using a specific count of symptoms as the best clinical cutoff point for arm lymphedema among breast cancer survivors, we explored the best clinical cutoff point for the sample in terms of count of symptoms, ie, the cutoff point of the count of lymphedema symptoms that maximized the sum of sensitivity and specificity. ${ }^{13,14}$ We used the freely available and open-source $\mathrm{R}$ program $^{15}$ for the examination of the best cutoff point for the sample according to Youden's method, that is, maximizing the sum of sensitivity and specificity. ${ }^{13,14}$ We used breast cancer survivors with lymphedema as the reference standard for calculation of sensitivity and specificity. Sensitivity rep- resents the rate of true positive cases found by the index test, while specificity represents true negative cases. Sensitivity and one minus specificity data over a range of lymphedema assessment outcomes were used to construct the receiver operating characteristic (ROC) curves, and area under the curve (AUC) was calculated with 95\% CIs. Higher AUC values represent greater diagnostic accuracy. ${ }^{16} \mathrm{An}$ AUC of 1.0 represents perfect sensitivity and specificity, while an AUC of 0.5 represents a test with weak sensitivity and specificity. ${ }^{16}$

\section{Results \\ Description of participants}

A total of 250 participants who completed the study were categorized into three study groups: 60 healthy female adults (24\%), 42 breast cancer survivors who had been previously diagnosed with lymphedema (16.8\%), and 148 at-risk breast cancer survivors $(59.2 \%)$. Participants in the three groups were similar in education and marital status. The healthy adults were significantly younger than the breast cancer survivors with lymphedema and at-risk survivors. Significantly more survivors in the lymphedema group were unemployed (Table 1). Significantly more nonwhite survivors had lymphedema. Survivors with lymphedema had significantly higher body mass index than women in the healthy and at-risk groups. More women in the lymphedema group had mastectomy, chemotherapy, and axillary lymph nodes dissection (Table 2).

Table I Participants' demographic characteristics $(n=250)$

\begin{tabular}{|c|c|c|c|c|c|c|c|}
\hline \multirow[t]{2}{*}{ Variable } & \multicolumn{2}{|c|}{ Lymphedema $(n=42)$} & \multicolumn{2}{|c|}{ At risk $(n=148)$} & \multicolumn{2}{|c|}{ Healthy $(n=60)$} & \multirow[t]{2}{*}{$P$-value } \\
\hline & Mean & SD & Mean & SD & Mean & SD & \\
\hline Age & 58.0 & 10.7 & 55.8 & 11.6 & 36.5 & 12.8 & $<0.001$ \\
\hline \multirow[t]{2}{*}{ Body mass index } & 28.8 & 7.11 & 25.5 & 4.99 & 26.9 & 7.12 & 0.02 \\
\hline & n & $\%$ & n & $\%$ & n & $\%$ & \\
\hline \multicolumn{8}{|l|}{ Education } \\
\hline Less than high school & 1 & 2.4 & 0 & 0 & 0 & 0 & 0.292 \\
\hline High school & 6 & 14.3 & 12 & 8.2 & 5 & 8.3 & \\
\hline Partial college & 8 & 19.0 & 29 & 19.7 & 17 & 28.3 & \\
\hline College graduate & 15 & 35.7 & 43 & 29.3 & 21 & 35.0 & \\
\hline Graduate degree & 12 & 28.6 & 62 & 42.2 & 17 & 28.3 & \\
\hline \multicolumn{8}{|l|}{ Marital status } \\
\hline Single/divorced/separated & 20 & 47.6 & 69 & 46.6 & - & - & 1.000 \\
\hline Married & 22 & 52.4 & 79 & 53.4 & - & - & \\
\hline \multicolumn{8}{|l|}{ Employment ${ }^{\mathrm{a}}$} \\
\hline No & 20 & 48.8 & 43 & 29.3 & 0 & 0 & 0.025 \\
\hline Yes & 21 & 51.2 & 104 & 70.7 & 60 & 100 & \\
\hline \multicolumn{8}{|l|}{ Race $^{a}$} \\
\hline White & 22 & 53.7 & 119 & 82.1 & 24 & 40.0 & $<0.001$ \\
\hline Nonwhite & 19 & 46.3 & 26 & 17.9 & 36 & 60.0 & \\
\hline
\end{tabular}

Note: a Missing data for one at-risk woman and one woman with lymphedema. Abbreviation: SD, standard deviation. 
Table 2 Clinical characteristics for breast cancer survivors $(n=190)$

\begin{tabular}{|c|c|c|c|c|c|}
\hline \multirow[t]{2}{*}{ Variable } & \multicolumn{2}{|c|}{$\begin{array}{l}\text { At risk } \\
(n=\mid 48)\end{array}$} & \multicolumn{2}{|c|}{$\begin{array}{l}\text { Lymphedema } \\
(n=42)\end{array}$} & \multirow[t]{2}{*}{$P$-value } \\
\hline & $\mathbf{n}$ & $\%$ & $\mathbf{n}$ & $\%$ & \\
\hline Mastectomy & 71 & 48.0 & 31 & 73.8 & $<0.01$ \\
\hline Lumpectomy & 97 & 65.5 & 16 & 38.1 & $<0.01$ \\
\hline Radiotherapy & 74 & 51.0 & 28 & 66.7 & 0.08 \\
\hline Chemotherapy & 67 & 45.3 & 34 & 81.0 & $<0.01$ \\
\hline $\begin{array}{l}\text { Sentinel lymph nodes } \\
\text { biopsy }\end{array}$ & 134 & 90.5 & 23 & 54.8 & $<0.01$ \\
\hline \multirow{2}{*}{$\begin{array}{l}\text { Axillary lymph nodes } \\
\text { dissection }\end{array}$} & 85 & 57.4 & 39 & 92.9 & $<0.01$ \\
\hline & Mean & SD & Mean & SD & \\
\hline $\begin{array}{l}\text { Number of nodes } \\
\text { removed }\end{array}$ & 8.0 & 8.7 & 19.4 & 11.9 & $<0.01$ \\
\hline $\begin{array}{l}\text { Years since cancer } \\
\text { diagnosis }\end{array}$ & 2.0 & 2.3 & 6.9 & 8.5 & $<0.01$ \\
\hline
\end{tabular}

Abbreviation: SD, standard deviation.

\section{Symptom profiles and validity}

Breast cancer survivors with lymphedema had significantly more lymphedema symptoms in comparison with the healthy adults and at-risk survivors. Only two symptoms, arm aching and limited shoulder movement, were reported by $6.7 \%$ of healthy female adults, and $<5 \%$ of healthy adults reported any one of the remaining symptoms. Healthy adult female participants had an average of zero symptoms in their dominant arms, while survivors with lymphedema had an average of ten symptoms and at-risk survivors reported an average of five symptoms (Table 3). The difference among the three groups in terms of the count of lymphedema symptoms was significant $(F(2,99)=117.53, P<0.001)$.

No participants in the healthy and lymphedema groups reported blistering in the dominant/affected arm. Fisher's exact test adjusted by the Bonferroni method demonstrated significant differences in all symptoms between the lymphedema and healthy groups. The most common symptoms reported by over $50 \%$ of survivors with lymphedema were

Table 3 Lymphedema symptoms

\begin{tabular}{lllll}
\hline Symptoms & Mean + SD & Median & \multicolumn{2}{l}{ Range } \\
\cline { 4 - 5 } & & & Min & Max \\
\hline Healthy adults & $0.50+1.58$ & 0 & 0 & 8 \\
At-risk survivors & $4.81+4.56$ & 4 & 0 & 20 \\
Survivors with lymphedema & $9.76+4.48$ & 10 & 1 & 18 \\
\hline
\end{tabular}

Notes: ${ }^{M}$ Mean scores differ significantly at $P<0.05$ between healthy and lymphedema group by the Tukey multiple comparisons of means; ${ }^{b}$ mean scores differ significantly at $P<0.05$ between lymphedema and at-risk group by the Tukey multiple comparisons of means.

Abbreviations: SD, standard deviation; Min, minimum; Max, maximum. arm swelling (100\%), arm tightness (71.4\%), arm heaviness (71.4\%), arm firmness (69\%), arm aching (61.9\%), tingling in the affected arm (59.5\%), limited arm movement (57.1\%), and arm tenderness (52.4\%). Apart from arm tenderness, survivors with lymphedema were significantly more likely to experience these symptoms than at-risk survivors. Also, significantly more survivors with lymphedema reported the following symptoms in comparison with at-risk survivors: stiffness in the affected arm (42.9\%), seroma formation (31\%), limited finger movement (26.2\%), increased arm temperature (19\%), and limited elbow movement (16.7\%). There were no significant differences between survivors with lymphedema and at-risk survivors in terms of the following symptoms: pain, numbness, burning, stabbing, soreness, arm redness, and blistering (Table 4).

Bivariate odds ratios were estimated to summarize the strength of association between the symptoms and lymphedema. The symptom of arm swelling, which was reported by $100 \%$ survivors with lymphedema, had significant odds ratio of $561(P<0.0001)$, while the symptom of blistering, which was reported by none of the survivors with lymphedema and none of the healthy adults, had insignificant odds ratio of $0.97(P=0.9853)$. Significant odds ratios $>2.0$ with a $P$-value $<0.05$ were found for all symptoms except stabbing, arm soreness, and blistering. Women who reported arm heaviness, arm firmness, increased arm temperature, seroma formation, tightness, limited arm movement, tingling, and arm aching had more than five times the odds of lymphedema compared to women without these symptoms, while women who reported limited finger movement, limited elbow movement, and limited wrist movement had more than four times the odds of lymphedema compared to women without these symptoms. Women who reported pain in the affected arm also had $>1.99$ times the odds of lymphedema in comparison with those who reported no pain in the affected arm (Table 5).

\section{Sensitivity and specificity}

Analysis of the ROC curve for the count of lymphedema symptoms as a continuous screening variable for discriminating between the lymphedema group and the healthy group yielded an AUC of 0.984 (95\% CI; $P<0.001)$. Analysis of the ROC curve for the count of lymphedema symptoms as a continuous screening variable for discriminating between the lymphedema group and the at-risk group yielded an AUC of 0.785 ( $95 \%$ CI; $P<0.001)$.

To discriminate breast cancer survivors with lymphedema from healthy women, the best diagnostic cutoff point for 
Table 4 Symptom profiles

\begin{tabular}{|c|c|c|c|c|c|}
\hline Symptoms & $\begin{array}{l}\text { Healthy } \\
(n=60) \\
n(\%)\end{array}$ & $\begin{array}{l}\text { At risk } \\
(n=I 50), \\
n(\%)\end{array}$ & $\begin{array}{l}\text { Lymphedema } \\
(\mathrm{n}=42) \\
\mathrm{n}(\%)\end{array}$ & $\begin{array}{l}\text { P-value, } \\
\text { lymphedema vs } \\
\text { healthy }\end{array}$ & $\begin{array}{l}\text { P-value, } \\
\text { lymphedema vs } \\
\text { at risk }\end{array}$ \\
\hline I. Arm swellinga,b & $\mathrm{I}(\mathrm{I} .7)$ & $26(17.3)$ & $42(100)$ & $<0.001$ & $<0.001$ \\
\hline 2. Limited shoulder movement $\mathrm{t}^{\mathrm{a}, \mathrm{b}}$ & $4(6.7)$ & $42(28.0)$ & $22(52.4)$ & $<0.001$ & 0.005 \\
\hline 3. Limited fingers movement $\mathrm{t}^{\mathrm{a}, \mathrm{b}}$ & $0(0)$ & $15(10)$ & II (26.2) & $<0.001$ & 0.011 \\
\hline 4. Limited arm movement $\mathrm{t}^{\mathrm{a}, \mathrm{b}}$ & $2(3.3)$ & $36(24)$ & $24(57.1)$ & $<0.001$ & $<0.001$ \\
\hline 5. Arm tenderness ${ }^{\mathrm{a}}$ & $\mathrm{I}(\mathrm{I} .7)$ & $71(47.3)$ & $22(52.4)$ & $<0.001$ & 0.603 \\
\hline 6. Pain in the affected arma & $2(3.3)$ & $60(40)$ & $19(45.2)$ & $<0.001$ & 0.596 \\
\hline 7. Arm firmness ${ }^{\mathrm{a}, \mathrm{b}}$ & $0(0)$ & $36(24)$ & $29(69)$ & $<0.001$ & $<0.001$ \\
\hline 8. Arm tightness $\mathrm{s}^{\mathrm{a}, \mathrm{b}}$ & $2(3.3)$ & $52(34.7)$ & $30(71.4)$ & $<0.001$ & $<0.001$ \\
\hline 9. Arm heaviness $\mathrm{s}^{\mathrm{a}, \mathrm{b}}$ & $2(3.3)$ & $23(15.4)$ & $30(71.4)$ & $<0.001$ & $<0.001$ \\
\hline 10. Numbness in the affected arm & $2(3.3)$ & $51(34.2)$ & $19(45.2)$ & $<0.001$ & 0.208 \\
\hline II. Stiffness in the affected arm & $3(5)$ & $33(22.1)$ & $18(42.9)$ & $<0.001$ & 0.010 \\
\hline 12. Arm achinga,b & $4(6.7)$ & $45(30)$ & $26(61.9)$ & $<0.001$ & $<0.001$ \\
\hline 13. Burning in the affected arm ${ }^{a}$ & $0(0)$ & $17(11.3)$ & $8(19)$ & $<0.001$ & 0.202 \\
\hline 14. Stabbing in the affected $\mathrm{arm}^{\mathrm{a}}$ & $0(0)$ & $27(18)$ & $10(23.8)$ & $<0.001$ & 0.507 \\
\hline 15. Tingling in the affected arm & $3(5)$ & $41(27.3)$ & $25(59.5)$ & $<0.001$ & $<0.001$ \\
\hline 16. Seroma formation ${ }^{a, b}$ & $0(0)$ & $10(6.7)$ & $13(31)$ & $<0.001$ & $<0.001$ \\
\hline 17. Arm soreness ${ }^{a}$ & $2(3)$. & $49(32.7)$ & $13(3 \mid)$ & $<0.001$ & 1.000 \\
\hline 18. Limited elbow movement ${ }^{\mathrm{a}, \mathrm{b}}$ & $0(0)$ & $9(6.1)$ & $7(16.7)$ & 0.001 & 0.052 \\
\hline 19. Limited wrist movement ${ }^{\mathrm{a}}$ & $0(0)$ & II (7.3) & $8(19)$ & 0.001 & 0.038 \\
\hline 20. Arm redness ${ }^{a}$ & $\mathrm{I}(\mathrm{I} .7)$ & $20(13.3)$ & $9(2 I .4)$ & 0.001 & 0.224 \\
\hline 21. Blistering in the affected arm & $0(0)$ & $2(1.3)$ & $0(0)$ & - & 1.000 \\
\hline 22. Increased arm temperature ${ }^{\mathrm{a}, \mathrm{b}}$ & $0(0)$ & $5(3.4)$ & $8(19)$ & 0.001 & 0.002 \\
\hline
\end{tabular}

Notes: ${ }^{a}$ Fisher's exact test followed by Bonferroni adjustment, significant difference between the lymphedema group and healthy group. The Bonferroni critical value

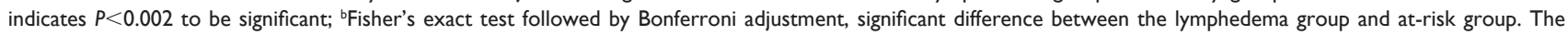
Bonferroni critical value indicates $P<0.002$ to be significant.

Table 5 Bivariate association between each symptom and lymphedema

\begin{tabular}{|c|c|c|c|}
\hline Symptom & $\begin{array}{l}\text { Odds } \\
\text { ratio }\end{array}$ & $95 \% \mathrm{Cl}$ & $P$-value \\
\hline I. Arm swelling & 561.00 & $76.04-71,644.49$ & $<0.0001$ \\
\hline 2. Arm heaviness & 17.46 & $8.22-39.25$ & $<0.0001$ \\
\hline 3. Arm firmness & 10.33 & $5.04-22.16$ & $<0.0001$ \\
\hline 4. Increased arm temperature & 9.07 & $2.98-29.94$ & $0.000 \mathrm{I}$ \\
\hline 5. Seroma formation & 8.61 & $3.54-21.54$ & $<0.0001$ \\
\hline 6. Arm tightness & 7.78 & $3.84-16.84$ & $<0.0001$ \\
\hline 7. Limited arm movement & 5.86 & $2.94-11.93$ & $<0.0001$ \\
\hline 8. Tingling in affected arm & 5.54 & $2.79-11.26$ & $<0.0001$ \\
\hline 9. Arm aching & 5.14 & $2.60-10.46$ & $<0.0001$ \\
\hline 10. Limited fingers movement & 4.56 & $1.92-10.66$ & 0.0008 \\
\hline II. Limited elbow movement & 4.39 & $|.53-| 2.2 \mid$ & 0.0069 \\
\hline 12. Limited wrist movement & 4.23 & $1.58-10.99$ & 0.0049 \\
\hline 13. Limited shoulder movement & 3.84 & $1.94-7.64$ & 0.0001 \\
\hline 14. Stiffness in the affected arm & 3.55 & $1.75-7.16$ & 0.0005 \\
\hline 15. Burning in the affected arm & 2.86 & I.II-6.93 & 0.0299 \\
\hline 16. Arm redness & 2.47 & $1.02-5.66$ & 0.0450 \\
\hline 17. Numbness in the affected arm & 2.40 & $|.21-4.7|$ & 0.0124 \\
\hline 18. Stabbing in the affected arm & 2.12 & $0.92-4.64$ & 0.0769 \\
\hline 19. Tenderness in the affected arm & 2.07 & $1.06-4.03$ & 0.0320 \\
\hline 20. Pain in the affected arm & 1.99 & $1.01-3.89$ & 0.0463 \\
\hline 21. Arm soreness & $\mathrm{I} .44$ & $0.68-2.92$ & 0.3285 \\
\hline 22. Blistering in the affected arm & 0.97 & $0.01-12.22$ & 0.9853 \\
\hline
\end{tabular}

Abbreviation: $\mathrm{Cl}$, confidence interval. count of lymphedema symptoms was three symptoms (AUC $=0.96$ [95\% CI: 0.95-0.98]; sensitivity $=0.94$ [95\% CI: 0.83-0.98]; specificity $=0.96$ [95\% CI: $0.88-0.99]$ ). To discriminate breast cancer survivors with lymphedema from at-risk survivors, the best diagnostic cutoff point was nine symptoms (AUC $=0.72$ [95\% CI: 0.64-0.80]; sensitivity $=0.64$ [95\% CI: $0.49-0.77]$; specificity $=0.80[95 \%$ CI: $0.73-0.86])$.

\section{Discussion}

Findings of our study provided updated evidence that breast cancer survivors continue suffering from multiple lymphedema symptoms in the ipsilateral upper extremity even with modern medical advances. All the survivors with lymphedema reported arm swelling. Significantly more survivors with lymphedema reported the following symptoms in comparison with at-risk survivors: arm tightness, arm heaviness, arm firmness, arm aching, tingling, limited arm movement, stiffness, seroma formation, limited finger movement, increased arm temperature, and limited elbow movement. Significant associations with lymphedema were found in bivariate logistic regression for all the symptoms, except 
stabbing, arm soreness, and blistering. While more research is needed to explore the etiology of lymphedema symptoms, physiologically, the accumulation of lymph fluid in the affected limb may create sensations of heaviness, tightness, and firmness and may also place undue pressure on nerves, producing feelings of aching and tingling. Accumulated lymph fluid in the affected arm also may result in stiffness and limited limb movement of arm, shoulder, fingers, and elbow. Increased temperature in the affected arm may be due to inflammation or cellulitis. Findings of our study provide preliminary evidence regarding the associations between symptoms and lymphedema.

Our study demonstrated that survivors with lymphedema have significantly more symptoms, indicating the potential use of symptom report for detecting lymphedema. We hypothesized that both healthy female adults and breast cancer survivors who had no lymphedema diagnosis and were never treated for lymphedema would have no or fewer lymphedema symptoms, while breast cancer survivors with lymphedema were expected to have significantly more lymphedema symptoms. These expectations were confirmed in our data for most women. Since all women who were treated for breast cancer are at life-time risk for lymphedema, using assessment methods that can identify lymphedema cases among at-risk breast cancer survivors is of the ultimate importance for clinical practice. Using a detecting cutoff of three symptoms, count of symptoms is able to discriminate breast cancer survivors with lymphedema and healthy participants. Using a detecting cutoff count of symptom of nine symptoms, count of symptoms is able to discriminate at-risk survivors and survivors with lymphedema. This is important since objective assessment of lymphedema, such as LVC and limb girth changes, might not be able to detect a latent stage of lymphedema, ${ }^{1,6,8,9}$ making count of symptoms a potentially useful early screening tool.

Perhaps, the most important contribution of the study was providing evidence that self-report of lymphedema symptoms has the ability to discriminate at-risk breast cancer survivors and those with lymphedema with fair sensitivity and specificity. Our study supports self-report of lymphedema symptoms as a time-efficient and cost-effective screening tool. It took $<5$ minutes for patients to complete the symptom assessment tool, and the one-page paper tool was inexpensive.

It should be noted that patients who experience symptoms or have a perception of lymphedema usually have an overall poorer quality of life. ${ }^{2,3,19}$ Although objective measures are considered superior to symptom assessment or patient's perception of lymphedema, perhaps in reality, from the patient's perspective, it is only the symptom experience and the perception of lymphedema that matter clinically because it is symptom experience and the perception of lymphedema that influence survivors' quality of life more than a measurement of interlimb volume or girth size.

\section{Strengths and limitations of the study}

The strength of the study lies on the very focus on patientcentered clinical outcome of self-reported lymphedema in detecting lymphedema. Low-cost and pragmatic self-care strategies, such as symptom report, may hold great promise for improving patients' quality of life. ${ }^{6,19,20}$ To date, as to our best knowledge, no research has been designed to evaluate the role of symptom report in detecting lymphedema among breast cancer survivors. In addition, the strengths of our study included well-defined contrast groups and the use of advanced statistical analyses.

Findings of the study should be considered in the light of the study limitations. The cross-sectional design limited the study's ability to prospectively examine the developments in lymphedema symptoms among the breast cancer survivors. Additional research is needed for utilizing a longitudinal design with baseline (prior to treatment) and consecutive follow-up assessments to capture any change during and after treatment in terms of lymphedema symptom experiences. It is possible that some at-risk survivors might have developed lymphedema that was not clinically apparent, since $>17 \%$ of at-risk survivors reported arm swelling and had reported more than nine symptoms and they were never diagnosed and treated for lymphedema.

\section{Conclusion}

Besides the fear of cancer recurrence, lymphedema and related symptoms are among the most important factors that elicit daily stress in breast cancer patients and negatively influence their quality of life. ${ }^{19}$ Cancer survivors face a lifelong risk of developing lymphedema since there is no defined period of time after cancer treatment when the risk no longer exists. ${ }^{18}$ Self-report of lymphedema symptoms is not only time-efficient but also cost-effective with fair sensitivity and specificity for discriminating at-risk breast cancer survivors and those with lymphedema. As a screening tool, the use of self-report of symptoms should be encouraged in clinical practice. Since early treatment usually leads to better clinical outcomes for this progressive and debilitating condition, ${ }^{1,2,20}$ in the absence of objective measurements capable of detecting latent stage of lymphedema, self-report of arm swelling 
and the count of symptoms may be a useful and cost-effective tool for early screening of lymphedema. Prospective research is needed to determine the predictive validity of symptom report with respect to subsequent development of breast cancer-related lymphedema.

\section{Acknowledgments}

This study was supported by the Avon Foundation, National Institutes of Health (NIMHD Project P60 MD000538-03), judges and lawyers for breast cancer alert, and the vital fund. The contents of this study are solely the responsibility of the authors and do not necessarily represent the official views of the NIH. The funders had no role in the study design, data collection, and analysis, decision to publish, or preparation of the manuscript.

\section{Disclosure}

The authors report no conflicts of interest in this work.

\section{References}

1. McLaughlin SA, Bagaria S, Gibson T, et al. Trends in risk reduction practices for the prevention of lymphedema in the first 12 months after breast cancer surgery. J Am Coll Surg. 2013;216:380-389.

2. Fu MR, Kang Y. Psychosocial impact of living with cancer-related lymphedema. Semin Oncol Nurs. 2013;29(1):50-60.

3. Fu MR, Ridner SH, Hu SH, Stewart BR, Cormier JN, Armer JM. Psychosocial impact of lymphedema: a systematic review of literature from 2004 to 2011. Psychooncology. 2013;22(7):1466-1484.

4. Fu MR, Rosedale M. Breast cancer survivors' experiences of lymphedema-related symptoms. J Pain Symptom Manage. 2009;38: 849-859.

5. Chachaj A, Małyszczak K, Pyszel K, et al. Physical and psychological impairments of women with upper lymphedema following breast cancer treatment. Psychooncology. 2009;19:299-305.

6. Cormier JN, Xing Y, Zaniletti I, Askew RL, Stewart BR, Armer JM. Minimal limb volume change has a significant impact on breast cancer survivors. Lymphology. 2009;42:161-175.
7. Fu MR, Chen CM, Haber J, Guth AA, Axelrod D. The effect of providing information about lymphedema on the cognitive and symptom outcomes of breast cancer survivors. Ann Surg Oncol. 2010;17(7):1847-1853.

8. Armer JM, Radina ME, Porock D, Culbertson SD, et al. Predicting breast cancer-related lymphedema using self-reported symptoms. Nurs Res. 2003;52(6):370-379.

9. International Society of Lymphology. The diagnosis and treatment of peripheral lymphedema. Lymphology. 2003;36:84-91.

10. McLaughlin SA, Wright MJ, Morris KT, et al. Prevalence of lymphedema in women with breast cancer 5 years after sentinel lymph node biopsy or axillary dissection: objective measurements. $J$ Clin Oncol. 2008;26(32):5213-5219. doi: 10.1200/JCO.2008.16.3725.

11. Hu S, Cleland CM, Kang Y. Fu MR. Measuring lymphedema symptom burdens: A psychometric study. The Multinational Association of Supportive Care in Cancer's annual meeting (MASCC/ISOO). In: 2012 International Symposium on Supportive Care in Cancer in New York City; June 28-30, 2012; New York.

12. Shi S, Lu Q, Fu MR, et al. Psychometric properties of the breast cancer and lymphedema symptom experience index: the Chinese version. Eur J Oncol Nurs. Epub 2015 Jun 9.

13. Youden WJ. Index for rating diagnostic tests. Cancer. 1950;3:32-35.

14. Perkins NJ, Schisterman EF. The inconsistency of "optimal" cutpoints obtained using two criteria based on the receiver operating characteristic curve. Am J Epidemiol. 2006;163(7):670-675.

15. Core Team R. R: A Language and Environment for Statistical Computing. Vienna, Austria: R Foundation for Statistical Computing; 2013. ISBN 3-900051-07-0, Available from: http://www.R-project.org/. Accessed July 13, 2015.

16. Smoot BJ, Wong JF, Dodd MJ. Comparison of diagnostic accuracy of clinical measures of breast cancer related lymphedema: area under the curve. Arch Phys Med Rehabil. 2011;92:603-610.

17. Welch BL. On the comparison of several mean values: an alternative approach. Biometrika. 1951;38:330-336.

18. Paskett ED, Naughton MJ, McCoy TP, Case LD, Abbott JM. The epidemiology of arm and hand swelling in premenopausal breast cancer survivors. Cancer Epidemiol Biomarkers Prev. 2007;16:775-782.

19. Fleissig A, Fallowfield LJ, Langridge CI, et al. Post-operative arm morbidity and quality of life. Results of the ALMANAC randomised trial comparing sentinel node biopsy with standard axillary treatment in the management of patients with early breast cancer. Breast Cancer Res Treat. 2006;95:279-293.

20. Fu MR, Axelrod D, Guth A, et al. Proactive approach to lymphedema risk reduction: a prospective study. Ann Surg Oncol. 2014;21(11): 3481-3498.
Breast Cancer: Targets and Therapy

\section{Publish your work in this journal}

Breast Cancer: Targets and Therapy is an international, peerreviewed open access journal focusing on breast cancer research, identification of therapeutic targets and the optimal use of preventative and integrated treatment interventions to achieve improved outcomes, enhanced survival and quality of life for the cancer patient.

\section{Dovepress}

View the full aims and scopes of this journal here. The manuscript management system is completely online and includes a very quick and fair peer-review system, which is all easy to use. Visit http:// www.dovepress.com/testimonials.php to read real quotes from published authors. 